\title{
Necesidad de origen y metafísica modal
}

\author{
José Tomás AlVARAdo Marambio \\ Instituto de Filosofía \\ Pontificia Universidad Católica de Chile \\ jalvaram@uc.cl
}

\begin{abstract}
Resumen: Kripke ha propuesto que el origen de una entidad debe ser concebido como esencial a él. El argumento de Kripke requiere una premisa crucial que establezca que cierto material en conjunto con una serie de condiciones no triviales deben ser suficientes para la realidad del objeto en cuestión. Sin embargo, los esfuerzos ulteriores de filósofos como Nathan Salmon o Graeme Forbes por hacer este requerimiento preciso y, al mismo tiempo, independientemente verosímil han fracasado. En este trabajo se explicará por qué esta línea de argumentación debe fracasar. El problema de la necesidad de origen requiere ser tratado de un modo sistemático diferente. Se argumenta que no existe un punto medio entre la necesidad de todas las condiciones de origen de un objeto y la contingencia de todas esas condiciones. En especial, la idea de que las condiciones de origen pueden ser necesarias sólo en su mayor parte, esto es, la posibilidad de admitir pequeñas variaciones en las condiciones de origen, es incoherente con una concepción sensata del dominio ontológico de lo modal.
\end{abstract}

Palabras clave: ontología, identidad, accesibilidad, principio de suficiencia

\begin{abstract}
Kripke proposed that the origin of an entity should be deemed as part of the essence of it. Kripke's argument required a crucial premise stating that certain hunk of matter and certain other non-trivial conditions are sufficient to deliver the entity in question. Further efforts by philosophers like Nathan Salmon and Graeme Forbes to make this requirement both precise and feasible have all nevertheless ended in failure. Here it is argued why this failure will always recur in these lines. The problem of the necessity of origin should be tackled in a different vein. It is argued that there seems to be no middle ground between necessity of all conditions of origin and contingency of all those conditions. Particularly, the idea that conditions of origin may be for the most part necessary, allowing the possibility of slight changes in them, is incoherent with a sensible conception of the ontological realm of the modal.
\end{abstract}

Key words: ontology, identity, accesibility, principle of sufficiency

Los conocidos trabajos de Saul Kripke ${ }^{1}$ y de otros filósofos de los años setenta han transformado la concepción semántica hasta ese momento prevalente y al mismo tiempo han provocado una intensa discusión sobre ciertas categorías conceptuales que parecían superadas hacía mucho tiempo. Kripke no sólo muestra que el significado de un nombre propio debe ser diferenciado de una descripción definida o de un racimo de descripciones, también señala que las nociones de necesidad/posibilidad deben ser distinguidas con cuidado de las nociones epistemológicas de $a$ priori/a posteriori y de analítico/sintético. Es difícil determinar ahora el impacto que estas precisiones habrán de provocar sobre el curso futuro del pensamiento filosófico, pero sí se puede afirmar que la influencia de las ideas de Kripke en los siguientes treinta años ha sido inmenso. La distinción

${ }^{1}$ Cfr. S. Kripke, Naming and Necessity, Harvard University Press, Cambridge, Mass., 1980. 
entre nociones modales y nociones epistémicas permitió inmediatamente hacer inteligibles conceptos metafísicos fundamentales para la tradición filosófica, como, por ejemplo, el concepto de 'esencia'. En efecto, si una atribución necesaria debe ser también una atribución a priori, esto es, si las nociones modales y las nociones epistémicas se conciben unidas, entonces el único modo de hacer inteligible la idea de que, por ejemplo, Micifuz es (esencialmente) un gato es suponiendo que tal idea ha de ser cognoscible a priori, ya sea que este conocimiento surja como resultado de alguna identidad conceptual tal que la proposición "Micifuz es un gato" resulte analítica, o bien que esta misma proposición resulte de uno u otro modo validada mediante un juicio sintético a priori. Las esencias postuladas por la tradición filosófica parecían entonces el más claro ejemplo del objeto de una pseudociencia que pretende iluminar la realidad de las cosas mediante meros análisis verbales. Por otro lado, nuestra concepción de que el conocimiento está constituido fundamentalmente por una reflexión inteligente sobre la evidencia empírica parece ser inmediatamente incompatible con la postulación de 'esencias' a las que se debe acceder a priori. Parecía surgir como un corolario inmediato de estas asociaciones que la ciencia empírica moderna no puede postular la existencia de esencias y que, por otro lado, el suponer que hay esencias de las realidades naturales es suponer que el filósofo pensando en su escritorio va a conseguir resultados más fructíferos para la iluminación de la naturaleza de la realidad que la que puedan obtener los investigadores de campo.

Afortunadamente, como se decía, esta lamentable dicotomía ha sido quebrada por los trabajos de Kripke, pues apareció ahora como idea plausible la de que algo necesario puede ser conocido a posteriori, esto es, por medios empíricos. Se hizo también plausible la idea de que la ciencia natural está buscando respuesta a la pregunta sobre qué son las entidades naturales y de que el modo de responder a esta pregunta es postulando 'esencias', que no tienen por qué ser conocidas a priori. Súbitamente, con las distinciones kripkeanas se ha iluminado un plexo de nociones metafísicas que parecían ya definitivamente desacreditadas. En este trabajo se pretende considerar con cierto detenimiento una de las sugerencias hechas por Kripke sobre el contenido de la esencia de una sustancia física: que para una sustancia física es esencial la materia de que ha sido originalmente compuesta y, en general, que para una sustancia material es esencial su origen. Esta sugerencia de Kripke sobre la esencia de una sustancia física en principio parece en conflicto con ciertas intuiciones modales generalmente aceptadas por el sentido común, para las que el material de que está compuesta una sustancia podría haber sido diferente o, al menos, podría haber sido levemente distinta sin afectar la identidad de la sustancia en cuestión. Posteriormente, las observaciones kripkeanas han sido profundamente elaboradas por la literatura filosófica de los años siguientes, y lo 
que era un argumento tentativo ha sido transformado en líneas de defensa relativamente bien trabadas. En lo que sigue, la reflexión sobre esas líneas de defensa y sobre las intuiciones modales de sentido común permitirá apreciar de qué modo nuestra concepción modal debe adoptar ciertas decisiones cruciales que se ponen de relieve en la discusión filosófica que será examinada.

\section{El argumento de Kripke}

Al considerar en Naming and Necessity la posibilidad de que una persona haya sido engendrada por otros padres y la posibilidad de que una mesa fuera hecha de otro material, Kripke señala:

Un principio sugerido por estos ejemplos es el siguiente: si un objeto material tiene su origen en cierta pieza de materia, no podría haber tenido su origen en ningún otro material. Algunas cualificaciones podrían ser necesarias (por ejemplo, la vaguedad de la noción de pieza de materia conduce a ciertos problemas), pero en una amplia clase de casos el principio es tal vez susceptible de algo así como una prueba, utilizando el principio de necesidad de la identidad para particulares. Sea 'B' el nombre (designador rígido) de una mesa, sea ' $A$ ' el nombre de la pieza de madera a partir de la cual actualmente viene. Sea ' $C$ ' el nombre de otra pieza de madera. Entonces supóngase que $B$ fuera hecha de $A$, como en el mundo actual, pero que simultáneamente también otra mesa $D$ fuera hecha de $C$. (Se supone que no hay ninguna relación entre $A$ y $C$ que hace que la posibilidad de hacer una mesa a partir de una dependa de la fabricación de una mesa a partir de la otra.) Ahora bien, en esta situación, $B \neq D$. Luego, incluso si $D$ fuera hecha por sí misma, y ninguna mesa hubiera sido hecha de $A, D$ no sería $B .^{2}$

El argumento de Kripke, que es explícitamente introducido de manera tentativa como algo que 'tal vez' podría ser considerado una prueba, funciona según el supuesto de que la identidad es necesaria. Esta tesis de la necesidad de la identidad fue uno de los elementos cruciales para la introducción de la distinción entre nociones modales y nociones epistémicas por la que Kripke es tan justamente célebre. Un argumento muy sencillo lleva a la conclusión de que si dos objetos son idénticos, entonces lo son de manera necesaria. Sea que:

${ }^{2}$ S. Kripke, Naming and Necessity, p. 114, n. 56. Kripke acota, además: "Hablando estrictamente, la 'prueba' usa la necesidad de la distinción, no de la identidad. Sin embargo, el mismo tipo de consideraciones que pueden ser usadas para establecer la última puede usarse para establecer la primera. (Supóngase que $X \neq Y$; si $X$ y $Y$ fueran ambas idénticas a un objeto $Z$ en otro mundo posible, entonces $X=Z, Y=Z$, luego $X=Y$.) Alternativamente, el principio se sigue de la necesidad de la identidad más el axioma 'brouweriano' o, equivalentemente, de la simetría de la relación de accesibilidad entre mundos posibles. En cualquier caso, el argumento se aplica sólo si la fabricación de $D$ desde $C$ no afecta la posibilidad de hacer $B$ de A, y viceversa." 
(1) $a=b$.

Vale el principio de Leibniz de indiscernibilidad de los idénticos, esto es:

(2) $\forall x \forall y((x=y) \rightarrow \forall P(P x \leftrightarrow P y))$.

Sea ahora la propiedad de ser necesariamente idéntico a ' $a$ ', esto es:

(3) $\lambda x \square(x=a)$.

Está claro que ' $a$ ' cae bajo la propiedad indicada en (3), pues:

(4) $\square(a=a)$.

Ahora bien, como ' $a$ ' es idéntico a ' $b$ ', por hipótesis, toda propiedad poseída por ' $a$ ' debe también ser poseída por ' $b$ '. Como ' $a$ ' posee la propiedad de ser necesariamente idéntico a ' $a$ ', se sigue que ' $b$ ' posee también la propiedad de ser necesariamente idéntico a ' $a$ ':

(5) $\square(b=a)$.

La conclusión, entonces, es que si ' $a$ ' y ' $b$ ' son idénticos, entonces deben ser idénticos de manera necesaria, esto es, en todo mundo posible se ha de dar tal identidad (en todo mundo posible, esto es, en el que exista la entidad en cuestión que se designa rígidamente como ' $a$ ' y también como ' $b$ '). Esto no quiere decir, naturalmente, que sea evidente desde el punto de vista epistemológico que ' $a$ ' es idéntico a ' $b$ '. El hecho de que el lucero de la mañana sea necesariamente idéntico al lucero de la tarde no impide que esta identidad haya sido un descubrimiento astronómico.

Se sigue de la necesidad de la identidad que si dos objetos son diferentes en algún mundo posible, entonces no son idénticos en ninguno. La necesidad de la identidad conlleva la necesidad de la diferencia. Supóngase, por hipótesis, que ' $a$ ' y ' $b$ ' fuesen diferentes para un mundo posible $w_{i}$ y fuesen idénticos para un mundo posible $w_{i i}$. Como la identidad es necesaria, se sigue que ' $a$ ' y ' $b$ ' también deben ser idénticos para $w_{i}$. Contradicción. Por ende, debe rechazarse la hipótesis. Los objetos ' $a$ ' y ' $b$ ', si son diferentes, son diferentes en todos los mundos posibles. ${ }^{3}$ El razonamiento de Kripke se apoya en esta tesis, pero prosigue de este modo: sea un objeto material $A$ compuesto por un trozo de materia $B$. Si el material de que está compuesto originalmente un objeto no es esencial a ese objeto, entonces $A$ podría haber sido compuesto por un trozo de materia diferente de $B$, que llamaremos $C$. Habría mundos posibles en los que $A$ está hecho de $C$ y no de $B$. Supóngase ahora que hay un mundo posible en el que $A$ está

\footnotetext{
${ }^{3} \mathrm{El}$ argumento supone que si $w_{i i}$ es accesible respecto de $w_{i}$, entonces $w_{i}$ es accesible respecto de $w_{i i}$. La relación de accesibilidad debe ser simétrica.
} 
hecho del trozo de materia $B$, pero que existe otro objeto material $D-$ semejante a $A$ en configuración- hecho de $C$. Como, por hipótesis, no es necesario que en todos los mundos posibles $A$ esté hecho de $C$, puede ser que mientras $A$ está hecho de $B$, el trozo $C$ constituye a $D$. Ahora bien, en este mundo posible $A \neq D$, luego en todos los mundos posibles $A$ y $D$ son diferentes. Si $D$ se compone de $C$, entonces parece que no podría ser que $A$ componga de ese mismo trozo $C$ en algún mundo posible. El razonamiento es generalizable para cualquier trozo de materia diferente de $B$, por lo que $A$ sólo puede estar compuesto de $B$.

Sin embargo, este argumento adolece de una dificultad fundamental. Dada la necesidad de la diferencia, esto es, la necesidad de la no identidad, si los objetos $A$ y $D$ se muestran diferentes en un mundo posible, deben ser diferentes en todos los mundos posibles. No obstante, sucede que el objetivo del argumento de Kripke no es simplemente mostrar que $A \neq D$, sino que la composición material de $A$, esto es, el trozo de materia $B$, es esencial a $A$. ¿Cómo es que del hecho de que $D$ sea diferente de $A$ se sigue que $A$ no pudo haberse compuesto de ningún otro trozo de materia diferente de $B$ ? La idea general del argumento es que cualquier trozo de materia alternativo que pudiese dar lugar a $A$ también daría lugar a un $D$ distinto de $A$; pero de esto no se sigue que $A$ esté necesariamente compuesto por un trozo de materia determinado $B$ si no es con la hipótesis de que un trozo de materia, como $B$ o $D$, no puede sino dar origen a un único objeto material. ${ }^{4}$ En efecto, supongamos que $A$ está compuesto en $w_{i}$ por el trozo de materia $B$ y en $w_{i i}$ por el trozo de materia $C$, y en $w_{i i i} A$ está compuesto de $B$ y existe además un objeto $D$ compuesto de $B$. Es efectivo que $A$ no puede ser idéntico a $D$, según lo muestra el argumento, pero en ningún caso se podría concluir —en virtud de este argumento- que el mundo posible $w_{i i}$ en que $A$ está compuesto de $C$ es ininteligible si no es suponiendo que a cada trozo de materia ha de corresponder un y sólo un objeto material compuesto de tal trozo de materia en todos los mundos posibles. Si se desecha tal principio, entonces es perfectamente inteligible (dejando entre paréntesis, naturalmente, la necesidad de origen sub iudice) que el mismo objeto $A$ en algunos mundos posibles esté compuesto de $B$, en otros mundos posibles, compuesto de $C$ y, en los mundos posibles en que exista otro objeto material diferente de $A$; naturalmente, deberá cuidarse de que los trozos materiales de que se componen dos objetos diferentes sean también diferentes. Todo lo que se requiere es que el trozo de materia $B$ dé lugar en algunos mundos posibles a $A$ y en otros mundos posibles no.

\footnotetext{
${ }^{4}$ Suponiendo que en el mundo posible en cuestión existe el trozo de materia. No se está diciendo que el trozo de materia exista en todos los mundos posibles, sino sólo que ese trozo de materia es suficiente para dar origen al objeto en cuestión y que en los mundos posibles en que el trozo de materia existe, también existe el objeto compuesto a partir de él.
} 
Considérese, además, que la idea de que un trozo de materia no está ligado a un único objeto material en todos los mundos posibles está muy afincada en nuestra comprensión ordinaria sobre qué es ser un objeto físico. Veo un jarrón de porcelana. La concepción de ese jarrón de porcelana como objeto físico es la de un trozo de material - la porcelana- a la que se ha dado una configuración peculiar tal que ese montón de material ha llegado a constituir dicho jarrón. Ese mismo montón de material podría haber dado origen a otros innumerables objetos físicos con otras formas si se tuviesen en vistas otras finalidades o hubiesen intervenido otros artífices provenientes de un contexto cultural diferente. Aun haciendo un jarrón, seguramente resultarán jarrones distintos de manos de un artesano chino que viviera bajo la dinastía Ming, de un indígena incaico del Cuzco o de un artesano francés del siglo XVII. Todos estos jarrones posibles están en la "potencia" de un mismo material y han de ser "educidos" de él mediante el trabajo y el designio creativo de un agente. El jarrón actual que veo es producto de la coincidencia de un mismo material y del proyecto y la eficiencia particulares de un agente.

Sucede, entonces, que el argumento de Kripke requeriría una premisa de este tenor:

(6) $\forall x \exists ! y \square((x$ es un trozo de materia) $\rightarrow$ (( $y$ es un objeto material) $\&$ $(y$ está compuesto de $x)))^{5}$

Este principio (6) parece obviamente falso y, por lo tanto, el argumento de Kripke no es suficiente para mostrar la necesidad de origen, tal como lo pretende.

\section{Reconstrucciones del argumento de Kripke}

La dificultad existente en el argumento de Kripke fue rápidamente percibida por Nathan Salmon ${ }^{6}$ y por otros intérpretes, como Graeme Forbes, ${ }^{7}$ que

${ }^{5}$ Aquí $\exists ! x$ es una abreviación de "existe un y sólo un $x \ldots$ ", esto es $\exists ! x F x \leftrightarrow$ $\exists x(F x \& \forall y(F y \rightarrow x=y))$. Debe considerarse, además, que en esta fórmula los cuantificadores se encuentran operando sobre un dominio constituido por todos los objetos posibles y no sólo los objetos actuales. Esta prevención vale para todas las restantes formulaciones de este trabajo. Por supuesto, la idea de "objetos posibles no actuales" requiere cierta explicación y elaboración filosófica y no puede simplemente darse por supuesta como coherente e inteligible sin más, pero tómese para los efectos de este trabajo simplemente como un recurso para hacer más perspicuas las formulaciones.

${ }^{6}$ Cfr. N. Salmon, Reference and Essence, 193-216. Salmon también expone su posición en "Modal Paradox: Parts and Counterparts, Points and Counterpoints", pp. 75-120; y "The Logic of What Might Have Been", pp. 3-34.

${ }^{7}$ De hecho, Forbes señala que él primero ideó un argumento de manera independiente para defender la necesidad de origen (cfr. G. Forbes, "Origin and Identity", pp. 353362) y sólo posteriormente se percató de que sus desarrollos estaban dirigidos a resolver 
propusieron formas de reparar la insuficiencia apuntada. En este trabajo, la discusión estará centrada fundamentalmente en las formas de reconstrucción de Salmon, pues permiten apreciar de manera más perspicua los rasgos sistemáticos fundamentales para una evaluación de la situación desde el punto de vista teórico. La dificultad fundamental que afecta la línea argumentativa desarrollada por Kripke radica en que no se sigue de que se haya mostrado que dos objetos materiales $A$ y $D$ son diferentes en un mundo posible que el trozo de materia que los constituye en tal mundo sea esencial a tales objetos, más que si se muestra, además, que tales trozos de materia —quizás con ulteriores cualificaciones que habrá que precisarsólo pueden dar origen a un único objeto en todos los mundos posibles. Hawthorne y Szabó Gendler resumen la línea argumentativa fundamental indicando que se requieren tres premisas en este tenor:

Suficiencia: si es posible que una entidad $r$ de tipo $k$ se origine de un trozo de materia $m$, entonces necesariamente cualquier entidad que se origine de $m$ y satisfaga ciertas restricciones (que llamaremos ' $C$ ') es $r$.

Imposibilidad: ninguna entidad puede originarse (en el mismo mundo) de cada uno de dos trozos de materia originante enteramente distintos (donde 'originarse' significa, entre otras cosas, "estar hecho enteramente de").

Composibilidad: si un trozo de materia $m$ puede llegar a constituir una entidad $r$ de tipo $k$ (satisfaciendo las restricciones $C$ ), y un trozo de materia posible disjunto ${ }^{8} n$ puede llegar a constituir una entidad de tipo $k$ (satisfaciendo las restricciones $C$ ), entonces existe un mundo posible en donde $m$ constituye una entidad $r$ de tipo $k$ y $n$ constituye otra entidad de tipo $k$ (satisfaciendo las restricciones $C$ en cada caso). ${ }^{9}$

Dadas estas premisas, se puede suponer, por hipótesis, qué es lo que acaece si la tesis de necesidad de origen es falsa. Sea que:

(7) El objeto $r$ está compuesto del trozo de materia $m$ en $w_{i}$.

la insuficiencia ya existente en el argumento kripkeano (cfr. "Origins and Identities", en http://www.tulane.edu/forbes/pdf_files/0\%261.pdf, especialmente la nota 2; este trabajo se ha incluido en A. Bottani et al. (comps.), Individuals, Essence and Identity. Themes of Analytic Metaphysics, Reidel, Dordrecht, 2001). Otros trabajos en los que Forbes desarrolla su posición son The Metaphysics of Modality, Clarendon, Oxford, 1985, pp. 132-159; "In Defense of Absolute Essentialism", pp. 2-31; "A New Riddle of Existence", pp. 415-430. Los argumentos de Forbes requieren un tratamiento separado para evaluarlos con justicia, lo que no puede hacerse aquí.

${ }^{8}$ Non-overlapping, esto es, se dice aquí que dos trozos de materia son "disjuntos" si no poseen ninguna parte en común.

${ }^{9}$ Hawthorne y Szabó Gendler, “Origin Essentialism: The Arguments Reconsidered”, p. 287. 
(8) El objeto $r$ está compuesto del trozo de materia $n$ en $w_{i i}$.

Si se supone, además, que los trozos de materia $m$ y $n$ son disjuntos, esto es, que no poseen ninguna parte en común, y además se supone que, cuando sea relevante, se satisfacen las condiciones $C$ asociadas, se sigue que:

(9) Existe un mundo posible $w_{i i i}$ en que: ( $m$ constituye una entidad de tipo $k) \&$ ( $n$ constituye una entidad de tipo $k$ ).

La proposición (9) se sigue del principio de composicionalidad. La existencia de los estados de cosas indicados en (7) y en (8) autoriza a postular un único mundo posible donde ambos estados de cosas se obtienen simultáneamente. Ahora bien, en virtud del principio de suficiencia y de (7) se sigue que en todo mundo posible en que algún objeto de tipo $k$ sea constituido por $m$, ese objeto debe ser $r$, y, además, en virtud de (8) se sigue que en todo mundo posible donde algún objeto de tipo $k$ sea constituido por $n$, ese objeto debe ser $r$. Luego vale para $w_{i i i}$ que:

( $r$ se origina de $m) \&(r$ se origina de $n)$.

Sin embargo, siendo tales trozos de materia disjuntos ex hypothesi, tal estado de cosas es imposible según lo declara el principio de imposibilidad. Un objeto material no puede estar enteramente compuesto de dos trozos de materia disjuntos en el mismo mundo posible (algo que quien rechace la necesidad de origen deberá aceptar). Por consiguiente, debe rechazarse la hipótesis de que un mismo objeto se constituya de diferentes trozos de materia en distintos mundos posibles.

Tal como se ha visto, el principio de imposibilidad —esto es, que no es posible que un mismo objeto esté compuesto de dos trozos de materia diferentes en el mismo mundo-y el principio de composicionalidad esto es, que si un objeto puede estar compuesto de un trozo de materia y otro trozo de materia puede dar origen a un objeto, entonces es posible que simultáneamente se den ambos objetos en un mundo- son ambos suficientemente plausibles. La dificultad tiene que ver con la formulación del principio de suficiencia. Debería existir cierto conjunto de condiciones y requerimientos tales que un mismo trozo de materia no pueda sino componer un mismo objeto en todos los mundos posibles, dadas tales condiciones. Ya se ha visto que un principio como el indicado en (6) es obviamente falso. La cuestión es, entonces, cómo cualificar tal principio con la introducción de las restricciones que sea menester de manera que (6) resulte verdadero y suceda que cierto trozo de materia $m$ sea no sólo necesario para constituir un objeto material $r$, sino que, además, sea suficiente para que exista $r$, dadas tales condiciones. Sea tal principio:

(11) $\forall x \exists ! y \square[((x$ es un trozo de materia) $\& C) \rightarrow((y$ es un objeto material) \& ( $y$ está compuesto de $x)$. 
La tarea de formular un argumento exitoso que muestre la necesidad de origen ha de radicar en la especificación del contenido de las condiciones $C$ tales que el principio enunciado en (11) resulte verdadero.

\section{Materiales y proyectos}

Tal como se ha enunciado ya, Salmon ha ofrecido una formulación de las condiciones $C$ requeridas para hacer verdadero el principio (11). Esta formulación fue objeto de refinamientos ulteriores para hacer frente a objeciones específicas. La primera formulación de tales condiciones $C$ es la siguiente, en el contexto del principio (11): ${ }^{10}$

(12) $\forall x \forall P \exists ! y \square[((x$ es un trozo de materia) \& ( $y$ ha sido construido de acuerdo con un plan $P)) \rightarrow((y$ es un objeto material) \& ( $y$ está compuesto de $x$ ))].

La idea de Salmon es que el "plan" en cuestión no sea simplemente un predicado sortal, en general, sino que sea una especificación de toda la configuración particular del objeto en cuestión, de tal manera que dos objetos que hayan sido construidos según el mismo "plan" — tomando "plan" en este sentido preciso- sean indiscernibles entre sí si no se considera el trozo de materia de que están compuestos y, presumiblemente, su localización espacio-temporal. Dos mesas construidas según el mismo "plan", entonces, serían dos mesas que no seríamos capaces de distinguir si cada una de ellas nos fuese presentada de manera sucesiva. Podemos distinguirlas si se presentan simultáneamente, porque podemos hacer una referencia indexical al lugar en que se encuentra cada una de ellas, pero no existen ni respectos cualitativos ni diferencias de forma que permitan distinguirlas. Se incluye, entonces, en el "plan" — tal como Salmon concibe esta noción- no sólo el predicado sortal con que se tipifica ese tipo de ente, esto es, no sólo la indicación de qué tipo de 'sustancia' es ese objeto, sino también todas sus determinaciones accidentales intrínsecas. Sería el "plan" del gato Micifuz, por ejemplo, el ser Micifuz un gato (y, por tanto, un felino, un mamífero, un vertebrado, un animal, etc.) y el ser de pelo jaspeado, de ojos verdes, de uñas largas, de cola de cinco centímetros, etcétera.

Salmon se ocupa de una objeción contra el principio de suficiencia que ha propuesto, recogido en (12), proveniente de un caso de modificación paulatina de partes materiales como se plantea en el famoso problema del barco de Teseo. ${ }^{11}$ Sea un objeto material cualquiera $r$ constituido por un conjunto de piezas materiales $m_{i}, m_{i i}, \ldots, m_{n}$. Este objeto posee un plan específico, de acuerdo con la noción de 'plan' estipulada por Salmon. Se

${ }^{10}$ Cfr. N. Salmon, Reference and Essence, pp. 205-211.

${ }^{11}$ Cfr. ibid., pp. 219-229. 
puede suponer, siguiendo el famoso ejemplo, que se trata de un barco, el mismísimo barco empleado por Teseo para viajar a Creta y librar a sus compatriotas atenienses del terrible Minotauro. Sucesivamente, cada una de las piezas $m_{i}, m_{i i}, \ldots, m_{n}$ es luego remplazada por otra diferente de la serie $n_{i}, n_{i i}, \ldots, n_{n}$ de manera que, al final del proceso, el barco de Teseo está compuesto no por las piezas $m_{i}, m_{i i}, \ldots, m_{n}$, sino por $n_{i}, n_{i i}, \ldots, n_{n}$. Luego se toman las piezas de las que originalmente estaba compuesto el barco de Teseo $m_{i}, m_{i i}, \ldots, m_{n}$ y se construye mediante ellas otro barco siguiendo exactamente el mismo plan del barco original. Esto normalmente se ha tomado como un experimentum crucis para cualquier concepción de la identidad de un mismo objeto que persiste en diversos instantes de tiempo. Aquí, sin embargo, Salmon tiene interés en el caso para considerar la viabilidad de su criterio de suficiencia recogido en (12). Es obvio que los barcos construidos mediante los materiales $m_{i}, m_{i i}, \ldots, m_{n}$ y $n_{i}, n_{i i}, \ldots, n_{n}$ y de acuerdo con el mismo plan no pueden ser el mismo barco. En el momento en que están los dos barcos construidos en ubicaciones diferentes, al mismo tiempo y con partes materiales constituyentes distintas no es de ningún modo plausible sostener que se trata del mismo barco. Pero sucede que el principio de suficiencia propuesto por Salmon y recogido en (12) lleva exactamente a esta conclusión increíble. En efecto, el barco construido inicialmente, sea $r$, estaba constituido en su origen por las partes materiales $m_{i}, m_{i i}, \ldots, m_{n}$ y según cierto plan determinado, sea $k$. El segundo barco, construido con las piezas desechadas de $r$, sea $s$, está constituido en su origen por las mismas partes materiales $m_{i}, m_{i i}, \ldots, m_{n}$ y el mismo plan $k$. El principio (12) dice que es suficiente, para que exista un objeto material, cierto material y un plan determinado. Aquí tanto el material como el plan son idénticos, luego $r$ y $s$ deben ser el mismo barco.

Salmon sugiere una reformulación del criterio de suficiencia recogido en (12) de manera que se excluya la ocurrencia de casos como el que se ha indicado. Señala Salmon:

[P]odemos reemplazar el principio $\left(V^{\prime}\right)$ [el equivalente al principio (12)] con el principio más débil de que si es posible para una mesa $x$ ser la única mesa construida originalmente (por cierto artesano en un determinado tiempo) a partir de cierto trozo de materia $y$ de acuerdo con cierto plan $P$, entonces necesariamente cualquier mesa que sea la única mesa construida originalmente (por el mismo artesano en el mismo lugar y al mismo tiempo) del mismo trozo $y$ de acuerdo con el mismo plan $P$ es la misma mesa $x$ y no otra. ${ }^{12}$

Entonces, la modificación propuesta por Salmon al criterio de suficiencia consiste en suponer que el objeto material constituido en un mundo posible de cierto trozo de materia y de acuerdo con un plan determinado,

${ }^{12}$ Ibid, p. 229. 
debe ser el único objeto constituido en ese mundo posible de ese material y según ese plan. Esto es, si se pretenden identificar dos objetos existentes en mundos posibles diferentes, será posible utilizar exitosamente el criterio prestado por el principio (12) sólo si en sus respectivos mundos posibles los objetos que van a ser comparados son el único objeto construido de cierto material y con un plan. En otras palabras, el criterio (12) funciona sólo si, por decreto, no hay en ese mundo casos de modificación paulatina de partes materiales como en el ejemplo del barco de Teseo. Una reformulación del principio en estas líneas quedaría de este modo:

(13) $\forall x \forall P \exists ! y \square[((x$ es un trozo de materia) \& ( $y$ ha sido construido de acuerdo con un plan $P$ ) \& (y es el único objeto construido de acuerdo con $P$ de $x)) \rightarrow((y$ es un objeto material $) \&(y$ está compuesto de $x)$ )].

Es preciso notar aquí varias dificultades que surgen con la reformulación. En primer lugar, debe considerarse que lo que estaría diciendo (13) es que para cualquier materia y cualquier plan, ocurre que hay un único objeto material constituido por tal materia y tal plan en todos los mundos posibles. Sin embargo esto es algo que está inscrito ya en la misma formulación del principio, pues el operador $\exists$ !, tal como se ha definido, dice precisamente eso. Parece, entonces, perfectamente ociosa la adición de la cláusula de unicidad propuesta por Salmon. La cuestión es que Salmon debe introducir esta restricción para la exclusión de los casos del tipo del barco de Teseo, esto es, simplemente para que el principio sea verdadero, pues, de otro modo, sencillamente no es verdadero que para todo trozo de materia y todo plan exista un único objeto material constituido a partir de ellos en todos los mundos posibles. La idea de la restricción impuesta es que el condicional vale en caso de que se satisfaga la identidad de plan y, además, sean excluidos todos los trucos de modificación paulatina de partes. Es razonable preguntar aquí si no es esto una reparación completamente ad hoc inmotivada por justificaciones independientes. Después de todo, lo que se espera de un principio de suficiencia viable es que indique en qué casos la existencia de cierto material es lo único requerido para que exista cierto objeto material, esto es, en qué condiciones $C$ sustantivas adicionales a la existencia de cierto trozo de materia se sigue que hay un objeto material determinado. La cláusula de unicidad introducida parece precisamente una adición no sustancial. En efecto, ¿qué es lo que diferenciaría la formulación (13) de esta otra?

(14) $\forall x \exists ! y \square[((x$ es un trozo de materia) \& ( $y$ es el único objeto construido de $x)) \rightarrow((y$ es un objeto material) \& (y está compuesto de $x)$ )]. 
Este supuesto principio de suficiencia estaría diciendo que la existencia de cierta materia es suficiente para que exista un único objeto material construido a partir de ella si ese objeto es, de hecho, el único objeto construido a partir de ella. Se trata de un principio verdadero, naturalmente, pero perfectamente trivial y carente de todo valor informativo. Considérese, por lo demás, que este principio (14) surge simplemente de quitar toda referencia a un plan, que era el factor que realizaba el grueso del trabajo para el principio de suficiencia de Salmon. Por lo tanto, el plan no realiza ninguna función teórica relevante si se introduce la cláusula de unicidad, pues la cláusula de unicidad en comento no hace sino introducir, por decreto, el cumplimiento del consecuente buscado. Sucede, entonces, que Salmon parece haber conseguido la corrección de su principio, pero sólo al precio de hacerlo por completo inofensivo e inservible.

Robertson ha propuesto una crítica al criterio de suficiencia de Salmon con un caso de un tipo "casi" barco de Teseo. ${ }^{13}$ Sea en $w_{i}$ un objeto material $r$ construido con el conjunto de trozos materiales $m^{\prime}$, que difiere sólo en unas pocas moléculas del trozo de materia $m$ y de acuerdo con el plan $k$. Paulatinamente se le quitan piezas a $r$ de manera que llegue a estar constituido por un conjunto de trozos de material ninguno de los cuales sea común a $m$. Luego, el material $m$ se toma y se constituye con él otro objeto material $s$ de acuerdo con el mismo plan $k$. Está claro que el objeto $r$ podría ser el primer objeto construido según el plan $k$, con el material $m$ (faltan para esto sólo unas pocas moléculas que cambiar en la materia originalmente constituyente de $r$ ), digamos que en el mundo posible $w_{i i}$, y sucede que $s$ también podría ser el primer objeto construido según $k$ con $m$. De hecho, esto es lo que sucede en el mundo posible $w_{i}$. En el mundo posible $w_{i i}$ en que $r$ es construido originalmente con $m$ de acuerdo con el plan $k$, el principio (13) señala que ese objeto es el mismo objeto que $s$ en $w_{i}$ construido de $m$ de acuerdo con el plan $k$. Pero tenemos que en $w_{i}$ sucede claramente que $r \neq s$. Como la inidentidad es necesaria, $r$ y $s$ no deberían ser identificados, pero esto es exactamente lo que hace el principio (13). Robertson estima que estos contraejemplos al principio (13) pueden generarse indefinidamente con tal de que se permitan pequeñas variaciones en la materia que constituye a un objeto físico. Sucede, sin embargo, que la posibilidad de variaciones leves en la materia que constituye originalmente a un objeto físico parece un principio modal verosímil.

$$
\begin{aligned}
& \forall x \forall y \exists z((x \text { es un objeto material) \& ( } x \text { está compuesto de } y)) \rightarrow \\
& \diamond((z \text { es levemente diferente de } y) \&(x \text { está compuesto de } z)) .
\end{aligned}
$$

${ }^{13}$ Cfr. T. Robertson, "Possibilities and the Arguments for Origin Essentialism", pp. 729-749. La crítica a que se hace referencia se encuentra en las pp. 737-738. Robertson agrega otros argumentos contra el criterio de suficiencia de Salmon a los que no se hará mención por parecer inconcluyentes. 
Salmon sólo podría defender la necesidad de origen al precio de rechazar este principio de flexibilidad (15), lo que hace tal necesidad de origen inverosímil en la misma medida en que el principio de flexibilidad (15) es verosímil.

En conclusión, parece que, por estrechas que sean las condiciones $C$ que se incorporen a un principio de suficiencia de la forma del principio (11), si es posible hacer pequeñas variaciones en el origen de un objeto material, será siempre posible refutar tal principio de suficiencia con un contraejemplo adecuado. La idea, entonces, es que la dificultad apuntada aquí por Robertson parece tener un valor sistemático general. ${ }^{14}$ Considérese que un principio de suficiencia debe poder establecer cuáles son las condiciones adicionales $C$ requeridas para que un trozo de materia $m$ dé lugar a un y sólo un mismo objeto material $r$ en todos los mundos posibles. Se supone que esta descripción debe ser lo más estrecha y ajustada posible de manera que no se produzcan errores en la identificación del objeto $r$. Si al mismo tiempo se admite un umbral de flexibilidad en el material de que se ha compuesto originalmente un objeto (como en las restantes condiciones de origen), entonces nada impide que — comoquiera que sean los estándares de exigencia impuestos por el principio de suficiencia- tales estándares sean violados. Las pequeñas variaciones admisibles pueden sumarse hasta hacer una gran variación que conduce a una aporía. Aquí los ejemplos pueden ser todo lo sofisticados que se quiera. Así, uno podría sentirse inclinado a ir acumulando restricciones en $C$ para cerrar todas las brechas posibles en las que se generen aporías, incluyendo no sólo el hecho de ser el único objeto construido a partir de un trozo de materia $m$ de acuerdo con un plan $k$, sino también el tiempo en que ha sido construido ese objeto, el artífice que lo ha fabricado (o su causa eficiente, en términos generales), el lugar donde se ha construido e incluso el puesto que ocupa en el orden de una serie de objetos producidos sucesivamente. Sea un principio de suficiencia fortalecido con la introducción —en las condiciones $C$ - del tiempo de construcción del objeto material en cuestión:

(16) $\forall x \forall P \forall t \exists ! y \square[((x$ es un trozo de materia) \& ( $y$ ha sido construido de acuerdo con un plan $P$ ) \& (y es el único objeto construido de acuerdo con $P$ en $t$ de $x)) \rightarrow((y$ es un objeto material) \& (y está compuesto de $x$ ))].

Considérese que, con esta adición del tiempo de construcción a las condiciones $C$ necesarias para la suficiencia del material en cuestión, se cierra la posibilidad al contraejemplo presentado por Robertson. En ese contraejemplo, hay un objeto $r$ compuesto de $m^{\prime}$ de acuerdo con el plan $k$ en el mundo

\footnotetext{
${ }^{14}$ Una intuición semejante también ha sido defendida por H. Noonan, "The Necessity of Origin", pp. 1-20.
} 
posible $w_{i}$. Las piezas materiales que componen a $r$ son sustituidas paulatinamente hasta que queda conformado por un trozo de materia completamente diverso de $m$. Ahora $m$ da lugar al objeto $s$ de acuerdo con el mismo plan $k$. En $w_{i}$, entonces $s$ es el único objeto construido de $m$ de acuerdo con el plan $k$. Sin embargo, sucede que, de conformidad con el principio (15), el objeto $r$ podría haber estado compuesto por un conjunto de trozos de materia levemente diferentes. Como $m^{\prime}$ difiere de $m$ sólo levemente, esto es, sólo en unas cuantas moléculas, parece perfectamente posible que $r$ hubiera estado compuesto por $m$ de acuerdo con el plan $k$. Hay un mundo posible $w_{i i}$ donde $r$ es el único objeto construido de $m$ de acuerdo con el plan $k$ y $r$ no sufre ningún proceso de sustitución paulatina de partes. Aquí el principio (13) identifica $r$ y $s$. Pues bien, con la cualificación que se ha introducido en el principio de suficiencia (16) parecería que esta dificultad se resuelve porque los tiempos en que son construidos $r$ y $s$ son diferentes. En $w_{i}$, donde son construidos tanto $r$ como $s, r$ es construido en el tiempo $t_{i}$ y $s$ es construido en un tiempo posterior $t_{i i}$. Ahora bien, en $w_{i i}$ el objeto $r$ es construido en el tiempo $t_{i}$. Luego, aunque coinciden el material $m$ y el plan $k$ de los objetos $r$ y $s$ en $w_{i i}$ y $w_{i}$, los tiempos de construcción son diferentes y, por lo tanto, de acuerdo con el principio (16), no cuentan como el mismo objeto.

La cuestión es que, por una razón sistemática de fondo, siempre es posible encontrar un contraejemplo adecuado, si se autoriza la suficiente flexibilidad de origen, tal como lo permite el principio (15). La intuición aquí es que un objeto material podría haberse construido en un tiempo levemente diferente del tiempo en que fue construido en el mundo actual (o en el mundo posible en cuestión). En el caso que se había considerado, $r$ fue construido en $t_{i}$ mientras $s$ fue construido en $t_{i i}$. Supóngase un mundo posible $w_{i}$ donde las cosas suceden exactamente igual como en el contraejemplo anterior, esto es, $r$ es construido en $t_{i}$ del material $m^{\prime}$ de acuerdo con el plan $k$. El material $m^{\prime}$ difiere sólo en unas pocas moléculas del material $m$. El objeto $r$ sufre un proceso de sustitución paulatina de partes tal, que $r$ llega a estar compuesto de un material totalmente diferente de $m$, sea $n$. En $t_{i i}$, con el material $m$ se construye el objeto $s$ según el plan $k$. Las condiciones de identidad del objeto $s$ son, entonces, el ser construido a partir del material $m$ de acuerdo con el plan $k$ en el tiempo $t_{i i}$. Ahora bien, $r$ podría haber sido construido a partir de un material levemente diferente, esto es, diferente sólo en unas cuantas moléculas, sea $m$. También sucede que $r$ podría haber sido construido en un tiempo levemente posterior a $t_{i}$, sea, por ejemplo, un segundo después de $t_{i}$, en $t_{i+1}$. Se va a suponer que la diferencia entre $t_{i}$ y $t_{i i}$ es de $n$ segundos. Pues bien, el objeto $r$ de $w_{i}$ construido de $m^{\prime}$ en $t_{i}$ es idéntico al objeto $r$ de $w_{i i}$ construido de $m$ en el tiempo $t_{i+1}$. Ahora considérese otro mundo posible $w_{i i i}$ donde se da el mismo objeto $r$ construido del material $m$ en el tiempo $t_{i+2}$. La diferencia entre 
el tiempo de construcción de $r$ en $w_{i i}$ y de $r$ en $w_{i i i}$ es de sólo un segundo, por lo que son el mismo objeto. Ahora bien, como la identidad es transitiva, si $r$-en- $w_{i i}=r$-en- $w_{i i i}$ y si $r$-en- $w_{i}=r$-en- $w_{i i}$, entonces se sigue que $r$-en$w_{i i i}=r$-en- $w_{i}$. Este procedimiento puede ser reiterado $n$ veces, hasta que lleguemos finalmente a un mundo posible $w_{n}$ donde $r$ ha sido construido del material $m$ de acuerdo con el plan $k$ en el tiempo $t_{i i}$ (recuérdese que $\left.t_{i i}=t_{i+n}\right)$. Sucede que este objeto $r$-en- $w_{n}$ es idéntico al objeto $s$ de $w_{i}$, pues están construidos del mismo trozo de materia $m$, según el mismo plan $k$, en el mismo tiempo $t_{i i}$ y son además el único objeto material en esos mundos posibles que satisfacen estas condiciones. Sucede también que, por transitividad de la identidad, $r$-en- $w_{n}=r$-en- $w_{i}$, pues los conecta una cadena de identidades todas ellas aceptables por los principios de flexibilidad en el material de origen y de flexibilidad en el tiempo. Pero sucede también que $r \neq s$, pues en $w_{i}$ son claramente diferentes y la inidentidad es necesaria. Resulta, entonces, una contradicción.

Entonces, la conclusión a la que esta discusión parece conducirnos es que la introducción del más leve principio de flexibilidad echará por tierra cualquier principio de suficiencia. Como tal principio de suficiencia cumple un papel fundamental para la argumentación de la necesidad de origen, ésta requeriría el compromiso con una tesis esencialista de un carácter especialmente fuerte y, para muchos, inaceptable. Robertson, en concreto, estima que éste es sencillamente un motivo para rechazar la necesidad de origen. En este trabajo, en cambio, dadas ciertas tesis relativas a la existencia de un dominio ontológico objetivo de lo modal, se utilizará esta misma incompatibilidad para desechar la posibilidad de pequeñas variaciones en las condiciones de origen. Pero antes de proseguir la consideración de esta cuestión convendrá discutir una maniobra de reparación del principio de suficiencia de Salmon propuesto por Hawthorne y Szabó Gendler. ${ }^{15}$ Estos autores señalan que una ulterior cualificación puede evitar la aporía que generan los casos de objetos compuestos por un material casi idéntico a otro. La maniobra consiste simplemente en prohibir tales materiales casi idénticos. Así, en el ejemplo que se ha considerado, el objeto $r$ se construye del trozo de materia $m^{\prime}$, siendo $m^{\prime}$ diferente de $m$ sólo en unas pocas moléculas. Sea un trozo de materia como $m^{\prime}$ una $m$-variante. Hawthorne y Szabó Gendler prohíben las $m$-variantes. Las condiciones $C$ requeridas para que funcione el principio de suficiencia deben incluir ahora de manera expresa que no exista ninguna $m$-variante del objeto en cuestión. El principio de suficiencia quedaría así:

(17) $\forall x \forall P \exists ! y \square[((x$ es un trozo de materia) \& ( $y$ se ha construido de acuerdo con un plan $P$ ) \& ( $y$ es el único objeto construido de acuerdo

${ }^{15}$ Cfr. Hawthorne y Szabó Gendler, "Origin Essentialism: The Arguments Reconsidered", pp. 288-291. 
con $P$ de $x$ ) \& $\neg \exists v$ ( $v$ está compuesto de un $x$-variante de acuerdo con $P)) \rightarrow((y$ es un objeto material) \& (y está compuesto de $x))$ ].

Por supuesto, este principio (17) es una reparación ad hoc que carece de motivaciones que lo hagan independientemente plausible. No obstante, aquí se dejarán estas dificultades a un lado para mostrar la profundidad del tipo de problemas sistemáticos apuntados por los contraejemplos del tipo considerado. Robertson, en particular, considera que un principio como (17) estaría sujeto a ciertos contraejemplos presentados por ella contra el principio de suficiencia de Salmon. ${ }^{16}$ No obstante, su objeción no es concluyente. Un contraejemplo del principio (17) puede ir, entonces, por otras líneas. Basta para esto que se permita flexibilidad en alguna de las condiciones de origen y que se considere algo análogo a una $m$-variante, pero que no tenga que ver con el material. En el ejemplo que se va a considerar ahora esta variante puede tener que ver con el tiempo en que ha comenzado a existir un objeto, que es una de sus condiciones de origen. Sea un mundo posible $w_{i}$ donde el objeto $r$ se compone de $m$ según el plan $k$ en el tiempo $t_{i}$. Es posible que $r$ se haya construido en un tiempo levemente diferente, sea un segundo después. Entonces existe un mundo posible $w_{i i}$ donde el mismo objeto $r$ está compuesto de $m$ de acuerdo con el mismo plan $k$, pero en el tiempo $t_{i+1}$. En este mismo mundo posible $w_{i i}$ el objeto $r$ sufre un proceso de sustitución paulatina de unas pocas de sus partes. Se unen las partes que se han sacado de $m$ más cuantas moléculas adicionales sean necesarias y con este trozo de materia, sea $m^{\prime}$, se construye otro objeto $s$ a partir de $m^{\prime}$ de acuerdo con el plan $k$. En $w_{i i}$ es claro que $r \neq s$. Nótese que este supuesto no está prohibido por la cláusula de unicidad, pues lo que tal cláusula prohibe es que en ese mundo posible exista otro objeto construido con el mismo material y el mismo plan, pero aquí $s$ no ha sido construido con el mismo material $m$, sino con $m^{\prime}$. Tampoco puede decirse que aquí $m^{\prime}$ sea una $m$-variante, pues para esto debería ser casi idéntica a $m$, pero sucede que sólo tiene unas pocas partes en común. Ahora bien, es posible que un objeto material se haya constituido de un material levemente diferente de aquel de que actualmente está compuesto. Sea, entonces, un mundo posible $w_{i i i}$ donde $s$ está compuesto de un

${ }^{16}$ Cfr. T. Robertson, "Essentialism: Origin and Order", pp. 299-307, en especial las pp. 301302. La objeción de Robertson es que hay un mundo posible $w_{i}$ donde $r$ está compuesto por $m$ de acuerdo con el plan $k$ y no hay ningún otro objeto en $w_{i}$ que satisfaga estas condiciones ni tampoco ningún otro objeto que haya sido construido de un material levemente diferente de $m$, esto es, no hay ningún otro objeto que haya sido construido de una $m$-variante. Por otro lado, hay un mundo posible $w_{i i}$ donde $s$ está compuesto por $m$ según el plan $k$ y es el único objeto que satisface tales condiciones y no hay objetos constituidos de $m$-variantes. El principio (17) identifica $r$ y $s$ y esto es visto por Robertson como problemático. Sin embargo, sucede que esto sólo puede resultar problemático si hay un mundo posible $w_{i i i}$ en que existan tanto $r$ como $s$ y conste, por lo tanto, que $r \neq s$, pero este mundo está prohibido por la cláusula de unicidad. La objeción de Robertson no es, por lo tanto, concluyente. 
material levemente diferente, sea $m_{i}$, de acuerdo con el plan $k$. No hay aquí $m_{i}$-variantes ni procesos de sustitución paulatina de partes. Está claro que $s$-en- $w_{i i i}=s$-en- $w_{i i}$. El siguiente paso es repetir este procedimiento de pequeñas variaciones $n$ veces, tal que se llegue finalmente a un mundo posible $w_{n}$ donde $s$ esté compuesto exactamente de $m$ de acuerdo con el plan $k$. Como $s$-en- $w_{n}$ está conectado por una serie de modificaciones aceptables según el principio de flexibilidad (15) con $s$-en- $w_{i i}$, se sigue que $s$-en- $w_{n}=s$-en- $w_{i i}$. Por lo demás, no hay en $w_{n}$ procesos de sustitución paulatina de partes ni $m$-variantes. Sucede, entonces, que el principio (17) identifica $r$-en- $w_{i}$ y $s$-en- $w_{n}$, pero consta que $r \neq s$ y la inidentidad es necesaria. Contradicción.

No parece, por lo tanto, que nuevas reformulaciones más ingeniosas puedan reparar de manera estable el principio de suficiencia de Salmon. Esto obliga a considerar la situación dialéctica desde una perspectiva diferente.

\section{La necesidad de origen y las relaciones de accesibilidad}

Tal como se ha podido apreciar, las líneas de argumentación desarrolladas hasta ahora para justificar la necesidad de origen adolecen de graves dificultades. La más grave de estas dificultades parece tener que ver con el hecho de que se ha pretendido justificar la necesidad de origen apelando, de manera expresa o tácita, a un principio de suficiencia prima facie inverosímil. El argumento de Kripke en Naming and Necessity requería de este principio por el que, dado un trozo de materia, se sigue que hay un único objeto producido con él. Como este principio es obviamente falso, al menos bajo esta formulación y sin ulteriores cualificaciones, la argumentación kripkeana no puede funcionar. La discusión posterior, especialmente en Salmon, ha tratado una y otra vez de argumentar a favor de la necesidad de origen reparando, en lo que cabe, el argumento original de Kripke, tratando de presentar principios de suficiencia que resulten más verosímiles. Hasta el momento, ninguna reparación ha funcionado.

En esta sección del trabajo se presentará una forma diferente de evaluar la cuestión de que la tesis de la necesidad de origen pueda ser comprendida en sus implicaciones filosóficas con más claridad y también pueda ser comprensible de mejor manera qué elementos de juicio favorecen una concepción metafísica general en la que el origen es parte de la esencia de un objeto. No se va a tratar, por lo tanto, de hacer nuevas reparaciones de la estrategia Kripke-Salmon con formulaciones más complejas del principio de suficiencia. Por el contrario, trabajará con el supuesto de que el principio es irreparable si las condiciones de origen no se especifican de manera completa. El sentido en que se entiende que una descripción de condiciones de origen es completa se precisará con detenimiento. 
El punto de partida que se adoptará aquí será simplemente considerar que las condiciones de origen de un objeto pueden ser o bien necesarias o bien contingentes. Si tales condiciones son necesarias, pueden ser o bien todas ellas necesarias o bien sólo algunas de ellas necesarias para la constitución de ese objeto. Debe hacerse notar aquí que cuando se habla de "objeto" se quiere indicar cualquier tipo de entidad de las que estimamos que existen en el espacio y en el tiempo, ya sean objetos materiales, organismos vivientes, vegetales, animales o agrupaciones de éstos, y que están sujetos a los procesos familiares de generación y corrupción. Se trata de una terminología que intenta ser, en lo posible, ontológicamente neutral sobre el modo de ser de tales entidades. No deben considerarse aquí entidades "abstractas" como números y entidades matemáticas, conjuntos o universales. Así, el tipo de objetos para los que significativamente puede valer o no valer la necesidad de origen es el de objetos que están compuestos de partes materiales y cuyo llegar a estar constituidos acaece en un instante de tiempo y en un lugar preciso del espacio. El llegar a ser o el generarse de un objeto de este tipo requiere, además de los elementos indicados, de una peculiar estructuración, forma o modo de disposición de las partes materiales entre sí. Estas cuestiones ya han sido consideradas de una u otra forma en las secciones anteriores, pero será conveniente volver a considerarlas aquí con detalle para delimitar con más precisión la cuestión que debe abordarse.

Entonces, las condiciones de origen de un objeto acerca del cual es relevante la cuestión de la necesidad de origen se componen de cuatro elementos: (a) un conjunto de trozos de materia de los que se compone el objeto (se designarán con las variables $m, n$ o con subíndices $m_{i}, m_{i i}, \ldots, m_{n}$ ); (b) cierta estructuración o forma en que se componen estas partes materiales (se designarán con las variables $k, j$ ); y (c) el tiempo y el lugar en que se produce el llegar a estar compuesto del objeto (lo que se designará con cuatro variables que representan coordenadas en el espacio-tiempo $x, y, z, t$; no se pretende con esto presuponer que el espacio-tiempo es una estructura ontológica unitaria). Ahora bien, ¿̇no se están aquí dejando a un lado otros factores que también pueden estimarse legítimamente como parte de las condiciones de origen de un objeto? ¿No podrían también incluirse en estas condiciones de origen el artífice del objeto, si se trata de un artefacto, o la finalidad de este artífice al producir el artefacto? La idea es que se debe entender que estos otros elementos se encuentran incluidos en la especificación de las condiciones de origen de un objeto o en los ancestrales de las condiciones de origen de un objeto de acuerdo con la enumeración que se ha hecho. En efecto, cuando, por ejemplo, se señala que son parte de las condiciones de origen de un objeto los trozos de materia que lo componen, está presupuesto que estos trozos de materia poseen condiciones de origen. Ese objeto que es el trozo de materia $m$, a su 
vez, está compuesto de un conjunto de trozos de materia $n$, configurados de acuerdo con una estructuración $k$ y que ha llegado a estructurarse tal como está en las coordenadas espacio-temporales $x, y, z, t$. El ancestral de la condición de origen de un objeto $r$ son las condiciones de origen de las condiciones de origen de $r$, o bien las condiciones de origen de las condiciones de origen de algo que en última instancia está conectado con $r$ por relaciones de este tipo. La relación que obtiene entre las condiciones de origen de un objeto $r$ y aquello a lo que ese objeto ha dado origen como una de sus condiciones de origen (por ejemplo, por ser una de sus partes materiales) es la relación de "dar origen a". Se dice que el par ordenado de objetos $\langle r, s\rangle$ se encuentra en la relación de 'dar origen a' si y sólo si $r$ es una condición de origen de $s$ o es un ancestral de una relación de origen de $s$.

Considérese, entonces, a un artífice de un objeto $r$. El artífice en cuestión fabrica el objeto $r$, por ejemplo, un auto, poniendo en funcionamiento la cadena de producción de una fábrica completamente automatizada. Puede suponerse que la cadena de producción está compuesta por autómatas, cada uno de ellos programados para tener una participación específica en el ensamblaje de las partes o para efectuar algún tipo de control de calidad en el proceso. Toda la contribución física del artífice ha sido dar la orden para que se desencadene el proceso conectando un interruptor. Todo lo restante funciona, por decirlo de algún modo, por sí solo. Puede también suponerse que el artífice se encuentra en un lugar muy distante de la fábrica; por ejemplo, el artífice es un niño africano instalado frente a su computadora en Sudáfrica, la fábrica está en algún lugar de Corea del Sur, la computadora está conectada a la internet y el modo específico como el niño sudafricano ha puesto en acción el mecanismo ha sido apretando suavemente el botón del ratón de su computadora. Dan origen, entonces, al automóvil $r$ todas sus condiciones de origen y todas las condiciones de origen de las condiciones de origen de $r$, lo que incluye las piezas materiales específicas $m$ de que está compuesto $r$, la estructuración $k$ en que viene dado $r$ y las coordenadas $x, y, z, t$ en que se ha producido el ensamblaje de todas estas partes. En las condiciones de origen de estas condiciones de origen de $r$ se incluyen, desde luego, la estructuración de las piezas que componen a cada uno de los autómatas que han contribuido a que $r$ llegue a tener la estructuración $k$ del conjunto de piezas $m$ desde $x, y, z, t \mathrm{y}$, en especial, las condiciones de origen del estado preciso de cada uno de estos autómatas en el momento en que hacían su contribución específica. Cada uno de estos estados puede definirse para cada instante de tiempo $t$, y las condiciones de origen de estos estados vienen dadas por el estado en el instante inmediatamente anterior a $t$. Así, pues, forman parte de las condiciones de origen de $r$ no sólo unas partes específicas estructuradas según la estructura abstracta $k$, sino toda la fábrica, todo el entorno de la fábrica, todo Corea del Sur y todo el mundo, incluyendo el estado del dedo del niño 
sudafricano en $x^{\prime}, y^{\prime}, z^{\prime}, t^{\prime}$, que despliega todo el proceso de producción al apretar el botón del ratón de su computadora. Ese apretar el botón ha sido parte de las condiciones de origen de un estado de ese ratón, y luego éste lo es de cierto evento eléctrico en unas moléculas que, a su vez, son condición de origen de otros eventos eléctricos en otras moléculas hasta llegar a la fábrica de Corea del Sur. Se puede decir legítimamente que el niño sudafricano da origen al automóvil $r$, pues es condición de origen de alguna condición de origen de algo que da origen a $r$. Se puede apreciar así que no es necesario introducir como condición de origen especial la aparición de un artífice o la intención que ha tenido ese artífice o un conjunto de artífices.

Sobre el estatus modal de estas condiciones de origen de un objeto $r$ cualquiera hay - tal como se ha indicado - tres opciones que pueden adoptarse prima facie sobre su necesidad. Es posible adoptar una concepción de necesidad de origen "fuerte" o una concepción de necesidad de origen "débil" o una concepción en la que las condiciones de origen son simplemente (todas ellas) contingentes. Será conveniente considerar, en primer lugar, qué dificultades afectan a una concepción de la necesidad de origen débil o "moderada", para luego determinar qué tipo de opción queda abierta entre la necesidad de origen fuerte y la contingencia de origen.

\section{1. La necesidad de origen débil}

Una concepción que a primera vista puede parecer razonable sobre la relevancia que pueden tener las condiciones de origen para la identidad de un objeto postularía que es esencial para un objeto el material de que ha sido compuesto, su forma de estructuración y el tiempo y lugar de generación, pero admitiendo pequeñas variaciones en tales condiciones de origen. Por ejemplo, no parece posible que Carlomagno hubiera podido nacer mil años después de cuando de hecho nació, ni tampoco que hubiese nacido en China como hijo de unos campesinos que viviesen a orillas del YangTse. Éstas son variaciones demasiado grandes en las condiciones de origen que resultarían intolerables para nuestras intuiciones modales sobre quién es Carlomagno. Sin embargo, parece admisible sostener que Carlomagno pudo haber sido engendrado dos minutos antes o dos minutos después del momento en que fue engendrado y, también, que pudo haber sido engendrado dos milímetros más hacia la derecha del lugar donde lo engendraron y que, en fin, pudo haber sido engendrado de un óvulo compuesto por tres moléculas diferentes de las que de hecho estaba compuesto el óvulo del que fue engendrado. La idea es que hay cierto margen o umbral de tolerancia para variaciones en las condiciones de origen de un objeto, sin que pueda considerarse que tales variaciones afecten a ese objeto en su esencia.

Sin embargo, esta idea presenta una dificultad inmediata consistente en que la suma de pequeñas variaciones puede constituir una gran variación; 
por ejemplo, supóngase que se estipulase en dos centímetros el umbral de tolerancia en el lugar de origen de un objeto. Así, el objeto $r$ constituido del trozo de materia $m$, de acuerdo con la forma $k$, en el tiempo $t$, en el lugar determinado por las coordenadas $x, y, z$, en el mundo posible $w_{i}$ contaría como idéntico al objeto $s$ constituido en el mundo posible $w_{i i}$, del mismo trozo de materia $m$, de acuerdo con la misma forma $k$, en el mismo instante de tiempo $t$, pero en el lugar determinado por las coordenadas $x, y, z+1,999$ centímetros. Sucede, entonces, que $r$-en- $w_{i}=s$-en- $w_{i i}$. Considérese ahora que $s$-en- $w_{i i}$ contaría, a su vez, como idéntico al objeto $u$ en el mundo posible $w_{i i i}$, constituido del mismo trozo de materia $m$, de acuerdo con la misma forma $k$, generado en el mismo instante de tiempo $t$, pero en el lugar determinado por las coordenadas $x, y, z+2,999$ centímetros. Se ve que la diferencia en el lugar de origen de $s$-en- $w_{i i}$ respecto del lugar de origen de $u$-en- $w_{i i i}$ es de sólo un centímetro y que no excede el umbral de tolerancia señalado de dos centímetros, por lo que $s$-en- $w_{i i}=u$-en- $w_{i i i}$. Por la transitividad de la identidad, se sigue que $r$-en- $w_{i}=u$-en- $w_{i i i}$, pero el lugar donde se han originado difiere en 2,999 centímetros, por lo que no pueden ser el mismo objeto. La diferencia en las condiciones de origen existente entre ambos constituye ya una diferencia esencial de acuerdo con el umbral que ha sido definido. Surge, por lo tanto, una contradicción. Este mismo tipo de aporías aparece también con el tiempo de generación, la estructuración de un objeto y el material de que está compuesto; esto es, con cualquiera de las clases de condiciones de origen definidas. Para la aporía no importa cómo sea la diferencia existente entre el margen definido por el umbral de tolerancia y las variaciones entre mundos posibles diferentes. Así, una variación permisible en el lugar de origen puede ser estipulada en veinte parsecs, y la diferencia entre cada uno de los mundos posibles considerados puede ser de tan sólo un milímetro. En este caso, habrá que acumular una cifra inmensa de mundos posibles para romper ese umbral, pero siendo la identidad una relación transitiva, esto no tiene ninguna relevancia.

Otra manera de apreciar el tipo de dificultades sistemáticas a que debe enfrentarse una concepción débil o moderada de la necesidad de origen aparece con la llamada paradoja de los cuatro mundos. ${ }^{17}$ Considérese que existen dos objetos en los mundos posibles $w_{i}$ y $w_{i i}$, respectivamente, que se diferencian sólo en algunos trozos de materia de que están compuestos. Cada uno de ellos, por ejemplo, está compuesto de cien piezas diferentes. El objeto $r$-en- $w_{i}$ está compuesto por los trozos de materia $m_{1}, m_{2}, \ldots, m_{97}, m_{98}, m_{99}, m_{100}$. Por otra parte, el objeto $s$-en- $w_{i i}$ está compuesto por los trozos de materia $m_{1}, m_{2}, \ldots, m_{97}, m_{101}, m_{102}, m_{103}$. Estos objetos $r$-en- $w_{i}$ y $s$-en- $w_{i i}$ comparten la misma estructuración $k$ y se han

${ }^{17}$ Cfr. N. Salmon, Reference and Essence, pp. 230-232. 
generado en las mismas coordenadas $x, y, z, t$. Se va a estipular que el umbral de tolerancia para variaciones en el material de que está compuesto un objeto es de $0.02(2 \%)$. Como $r$-en- $w_{i}$ y $s$-en- $w_{i i}$ difieren en tres piezas, hay que postular que $r$-en- $w_{i} \neq s$-en- $w_{i i}$. Sucede ahora que $r$-en$w_{i}$ es idéntico al objeto $r$ en el mundo posible $w_{i i i}$ que comparte la misma estructuración $k$, que ha sido generado también en $x, y, z, t$ y que está compuesto de los trozos de materia $m_{1}, m_{2}, \ldots, m_{97}, m_{98}, m_{102}, m_{103}$. En efecto, $r$-en- $w_{i}$ y $r$-en- $w_{i i i}$ difieren sólo en dos piezas, lo que se encuentra dentro del umbral de tolerancia para variaciones en la composición material. Por otra parte, $s$-en- $w_{i i}$ es idéntico al objeto $s$ en el mundo posible $w_{i v}$, que posee la misma estructuración $k$, ha sido generado en las mismas coordenadas $x, y, z, t$ y que está compuesto de los trozos de materia $m_{1}, m_{2}, \ldots, m_{97}, m_{98}, m_{102}, m_{103}$. En efecto, $s$-en- $w_{i i}$ y $s$-en- $w_{i v}$ difieren sólo en una pieza, lo que se encuentra dentro del umbral de tolerancia para variaciones en la composición material. En este punto surge la paradoja, pues se tiene que $r$-en- $w_{i}=r$-en- $w_{i i i}, s$-en- $w_{i i}=s$-en- $w_{i v}$ y $r$-en- $w_{i} \neq s$-en- $w_{i i}$. De aquí se sigue que $r$-en- $w_{i i i} \neq s$-en- $w_{i v}$, pero éstos coinciden exactamente en la misma estructuración $k$, han sido generados en las mismas coordenadas $x, y, z, t$ y están compuestos por el mismo conjunto de piezas materiales $m_{1}, m_{2}, \ldots, m_{97}, m_{98}, m_{102}, m_{103}$. En otras palabras, no existe entre ellos ninguna diferencia relevante y, sin embargo, el escenario de cuatro mundos que se ha presentado obligaría a postular que en realidad son diferentes.

¿Qué debe concluirse a partir de estas paradojas? El resultado paradójico obliga a hacer una revisión de las premisas que han conducido al resultado inaceptable. En los dos casos presentados se han utilizado supuestos muy sencillos: la transitividad de la identidad, cierta tolerancia con un umbral de variaciones permisibles en las condiciones de origen y una tesis modal característica de sistemas modales del tipo S-4 según la cual si algo es posiblemente posible, entonces es posible. En efecto, la primera paradoja surge porque, si se admite un umbral de tolerancia para pequeñas variaciones en las condiciones de origen, será siempre posible mostrar un mundo posible suficientemente lejano donde tal umbral será roto, pero que estará conectado por una cadena de identidades todas ellas permisibles de acuerdo con el umbral de tolerancia definido. La segunda paradoja surge porque dos objetos que resultan diferenciados según cierto umbral de tolerancia pueden mostrarse, a su vez, como respectivamente idénticos a objetos que son idénticos entre sí según todos nuestros parámetros, o según todos los parámetros que se quieran estipular para decidir la cuestión. Pues bien, ¿cuál de estos supuestos debe ser rechazado?

Parecería que la solución más razonable es sencillamente rechazar la hipótesis de algún margen de tolerancia para las condiciones de origen, por pequeña que esta variación sea. No obstante, hay otras formas de concebir la naturaleza de la modalidad, en general, y las relaciones que pueden 
darse entre los objetos de mundos posibles diferentes en las que no surgen de ningún modo estas dificultades. Si los individuos sólo pueden existir en un mundo posible y las relaciones que pueden tener con individuos en otros mundos posibles consisten simplemente en la relación de ser más o menos semejantes (y, de acuerdo con el contexto en que estas semejanzas y diferencias sean evaluadas, pueden calificarse como "contrapartidas"), entonces no puede aparecer ninguna paradoja, pues jamás es el caso que objetos ubicados en mundos posibles diferentes sean identificados como "el mismo" objeto. ${ }^{18}$ Esta alternativa radical no será considerada aquí, aunque no carece de interés sistemático. Se va a suponer que hay objetos idénticos en varios mundos posibles y que está idea está presupuesta en nuestras formas de razonamiento contrafáctico por las que se considera lo que hubiera sucedido si, por ejemplo, Carlomagno no hubiese sido coronado emperador en la Navidad del año 800. Si se desatiende esta concepción metafísica general alternativa, entonces las opciones sistemáticas que quedan por considerar son, por un lado, la mera contingencia de las condiciones de origen $\mathrm{y}$, por otro lado, poner en entredicho las concepciones sobre la identidad y la modalidad que están presupuestas en las argumentaciones que han conducido a los resultados aporéticos. ${ }^{19}$

Ha habido filósofos que, considerando esta clase de aporías y, por otro lado, estimando que son irrenunciables las intuiciones ordinarias sobre la posibilidad de un umbral de tolerancia para variaciones en las condiciones de origen, han creído que el modo más razonable como deben tratarse tales aporías es mostrando la naturaleza profunda de la identidad y de la

\footnotetext{
${ }^{18}$ Es lo que sucede, naturalmente, en la concepción metafísica de la modalidad de D. Lewis, "Counterpart Theory and Quantified Modal Logic", pp. 26-46; On the Pluralilty of Worlds, especialmente en las pp. 210-220.

${ }^{19} \mathrm{~N}$. Salmon también sostiene que las aporías surgen en parte por la operación implícita de una concepción ontológica fisicalista, por la que toda la entidad de los entes viene dada por los materiales de que están compuestos y su modo de estructuración (cfr. Reference and Essence, pp. 237-238). No se dejaría espacio para, por ejemplo, cierta haecceitas primitiva, por la que la identidad de un objeto está dada por algo más que la suma de las propiedades intrínsecas que ese objeto instancia. Hay que hacer notar que, dejando a un lado la viabilidad que puedan tener concepciones modales de cierta haecceitas primitiva, la dificultad apuntada por Salmon tal vez podría aliviar la paradoja de los cuatro mundos (u otras análogas), pero no es capaz de aliviar la situación teórica generada por los casos mucho más sencillos —de tres mundos-, donde una asignación de identidades choca directamente con una tesis de necesidad de origen débil. En el caso de la paradoja de los cuatro mundos, se aliviaría el problema porque se podría sostener — si se atiende a la sugerencia de Salmon-que aunque los objetos $r$-en- $w_{i i i}$ y $s$-en- $w_{i v}$ comparten el mismo material de que están compuestos, la misma estructuración y las mismas coordenadas de generación, en realidad son distintos, tal como lo requiere el escenario de cuatro mundos. En el caso más simple de una cadena de identidades que rompe el umbral de tolerancia asignado, por el contrario, el único modo de aliviar la situación sería rechazando de plano la inteligibilidad de la necesidad de origen débil (que es la posición que aquí se favorece) o modificando la naturaleza de la identidad o de las modalidades que operan en el escenario.
} 
modalidad metafísica. Ésta, en especial, ha sido la forma en que la cuestión ha sido abordada por Nathan Salmon. ${ }^{20}$ Considérese de nuevo la primera aporía que se indicó. El objeto $r$-en- $w_{i}$ es idéntico al objeto $s$-en- $w_{i i}$, y el objeto $s$-en- $w_{i i}$ es idéntico al objeto $u$-en- $w_{i i i}$. Si se concluye de esto que $r$-en- $w_{i}$ es idéntico a $u$-en- $w_{i i i}$, entonces debe desatenderse el umbral de tolerancia definido por el cual eran admisibles variaciones en el lugar de origen de no más de dos centímetros, pues entre $r$ y $s$ existe una variación en el lugar de origen de 2,999 centímetros. Debe aquí recordarse que, cuando se están definiendo estos umbrales de tolerancia para las condiciones de origen de un objeto, lo que se está tratando de especificar de una manera precisa es qué clase de vicisitudes o accidentes pueden sucederle a un mismo objeto en situaciones posibles de tal manera que legítimamente se pueda seguir hablando de vicisitudes que acaecen al mismo objeto y no a un objeto relativamente semejante por un conjunto de respectos. Un margen de tolerancia para las condiciones de origen determina qué circunstancias determinantes del origen de un objeto podrían haber sido diferentes sin que ese objeto hubiese dejado de ser ese mismo objeto. La situación posible en la que un mismo objeto posee condiciones de origen diferentes puede concebirse como un mundo posible donde el mismo objeto está sujeto a circunstancias que varían respecto de las circunstancias a las que, de hecho, tal objeto se ha visto realmente sometido en el mundo actual. Lo que surge de este examen es la esencia de ese objeto individual que podría definirse como el conjunto de propiedades intrínsecas que ese objeto satisface simultáneamente en todos los mundos posibles en los que existe. Entonces, si el objeto $r$-en- $w_{i}=s$-en- $w_{i i}$, lo que se está sosteniendo es que la situación descrita en la que un objeto, sea $s(=r)$, se genera en el lugar señalado por $x, y, z+1,999$ centímetros es posible respecto de $w_{i} \mathrm{o}$, como también suele decirse, es accesible desde $w_{i}$. Hay sistemas lógicos en los que la relación de accesibilidad entre mundos posibles no es transitiva, por lo que no es una ley lógica generalmente válida la tesis:

$$
\diamond \diamond p \rightarrow \diamond p
$$

Es precisamente una tesis como (18) la que se requiere para que puedan funcionar los argumentos que conducen a las aporías que se han indicado. ${ }^{21}$ En las aporías, lo que causa las dificultades es que algo que es posible respecto de un mundo posible con el que el mundo actual (o el que sea que se esté empleando como referente) guarda una relación de accesibilidad, es sencillamente accesible respecto del mundo actual. Si es posible, por ejemplo, respecto de $w_{i i}$ que el objeto $s$ hubiese sido generado en un lugar con un centímetro de diferencia (esto es, $u$-en- $w_{i i i}$ ), y es antes accesible ese

${ }^{20}$ Cfr. N. Salmon, Reference and Essence, pp. 238-240.

${ }^{21}$ Esta tesis (18) se sigue directamente del axioma característico de un sistema modal $S 4 \square p \rightarrow \square \square p$, mediante contraposición y sustituyendo luego $-p$ por $p$. 
mundo posible $w_{i i}$ respecto de $w_{i}$ (esto es, es posible que el objeto $r$ hubiese sido generado en un lugar que varía en 1.999 centímetros de donde fue de hecho generado), entonces $w_{i i i}$ debe ser accesible respecto de $w_{i}$. Esta relación de accesibilidad rompe inmediatamente el margen de tolerancia que había sido definido y se genera la paradoja, pues se estaría admitiendo que el objeto $r$-en- $w_{i}$ podría haberse generado en un lugar 2,999 centímetros diferente, cuando el umbral de tolerancia definido sólo admitía una diferencia de dos centímetros a lo más. Pues bien, la estrategia de Salmon es preservar la idea de que existen márgenes de tolerancia para variaciones en las condiciones de origen de un objeto, aunque esta intuición tenga el costo de obligar a rechazar que las relaciones de accesibilidad entre mundos posibles sean transitivas. Si se preserva esta intuición, entonces debe sostenerse que las leyes lógicas que rigen los enunciados modales que pueden formularse significativamente sobre la realidad no son las contenidas en sistemas como $K 4$ (en el que operan relaciones de accesibilidad transitivas), $S 4$ (en el que operan relaciones de accesibilidad reflexivas y transitivas) o $S 5$ (en el que operan relaciones de accesibilidad reflexivas, simétricas y transitivas).

\subsection{La necesidad de origen fuerte}

La cuestión crucial que debe dilucidarse en relación con la necesidad de origen, según se desprende de todo el examen que se ha realizado, tiene que ver con un balance de dos intuiciones que parecen conducir a conclusiones contrapuestas y que no son compatibles: por un lado, la intuición de que deberían ser admisibles pequeñas variaciones en las condiciones de identidad de un objeto sin que la identidad de tal objeto se vea afectada; por el otro, la intuición de que las relaciones modales de accesibilidad entre mundos posibles deben ser reflexivas, simétricas y transitivas. Si se concede más peso a la primera intuición, entonces uno se inclinará por una concepción débil de la necesidad de origen; en cambio, si se concede más peso a la segunda intuición, se estará más inclinado por una concepción fuerte de la necesidad de origen. En esta sección se tratará de clarificar qué tipo de motivaciones subsisten detrás de cada una de estas opciones sistemáticas.

Considérese en primer lugar qué razones pueden motivar el quiebre de la transitividad de las relaciones modales de accesibilidad. Es obvio que la motivación central detrás de posturas como la de Salmon es la pretensión de defender la intuición generalmente aceptada de que deben admitirse pequeñas variaciones en las condiciones de origen sin afectar la identidad. Esta intuición descansa en el sentido común o en la intuición modal sobre en qué es lo que consiste el ser de una entidad material para el común de nuestros pares culturales. Sin embargo, también es obvio que esta intuición generalmente aceptada no basta para la justificación de una 
tesis metafísica. Tal intuición puede tomarse como un punto de partida para la discusión metafísica sobre la cuestión, pero nuestra concepción metafísica de la realidad no es simplemente la concepción del vulgo puesta por escrito. El sentido común también ha creído perfectamente razonable que el sol gira en torno a la Tierra y que las brujas vuelan en escoba para celebrar sus aquelarres cada plenilunio, pero estas creencias con el tiempo han mostrado ser falsas o incoherentes. En otras palabras, la sensibilidad filosófica del hombre de la calle no es sacrosanta y no debe estimarse como inmune a la crítica racional. Es más, esta criba racional de las intuiciones del sentido común ha sido gran parte de la tarea social del filósofo.

Sucede, entonces, que el hecho de que cierta intuición se encuentre generalmente aceptada no es motivo suficiente para estimar justificada dicha intuición desde un punto de vista racional o, al menos, no es motivo suficiente si es que tal intuición no se encuentra acreditada por otros elementos que aporten evidencia a su favor. Creo, sin embargo, que hay muy buenos motivos para pensar que la intuición de sentido común es incoherente en este punto preciso de la necesidad de origen y que haríamos bien en reformarla. Puede interpretarse que las aporías muestran el quiebre de los umbrales de tolerancia postulados y precisamente ponen de manifiesto esta incoherencia dado el carácter de las nociones y de los principios modales y, también, dado el carácter de los hechos modales a los que tales conceptos y principios responden.

Ha sido una de las aportaciones fundamentales de Kripke haber mostrado que los aspectos metafísicos relativos a la modalidad deben deslindarse cuidadosamente de los aspectos epistémicos. Lo necesario no es idéntico a lo analítico desde un punto de vista lógico ni a lo a priori, desde un punto de vista epistemológico. Con las precisiones hechas por Kripke, las nociones ontológicas de 'esencia' y de 'propiedad necesaria' se han hecho plausibles y han llegado a integrar la visión mejor acreditada sobre el tipo de estructuras ontológicas que son objeto de la investigación científica. La necesidad y la posibilidad que están fundadas en la "esencia" o "naturaleza" de las entidades naturales poseen un carácter enteramente ontológico. En otras palabras, no se trata de lo que "debe" suceder o de lo que "puede" suceder dadas nuestras capacidades de imaginar escenarios diferentes del actual, sino de lo que "debe" o "puede" suceder dada la configuración ontológica real que se encuentra operativa en los dinamismos naturales. La necesidad metafísica u ontológica y, correlativamente, la posibilidad metafísica u ontológica no deben concebirse como dependientes en forma alguna de nuestras capacidades o aptitudes para concebir o llegar a conocer las estructuras de lo real, sino dependientes de las estructuras de lo real postuladas con existencia objetiva y con completa independencia de nuestra contribución mental, o, en términos generales, con completa independencia de nuestra contribución cognoscitiva. 
Esta concepción de la necesidad metafísica será objetable para filósofos de inclinación más o menos antirrealista, esto es, para filósofos que estén inclinados a pensar que el valor de verdad de nuestras proposiciones (o de las proposiciones modales, específicamente) es dependiente de nuestras capacidades cognoscitivas para verificar o refutar tales proposiciones, como también será objetable para filósofos que estén inclinados a pensar que sólo son concebibles objetos y propiedades realmente existentes dentro de un esquema conceptual de nuestra invención. No es el propósito de este trabajo abordar tales concepciones de manera general; estas alternativas se pondrán entre paréntesis en el desarrollo que sigue. La cuestión que se quiere argumentar aquí es que si es inteligible la idea de cierto ámbito ontológico objetivo de "hechos modales", entonces no hay espacio para la inteligibilidad de una necesidad de origen débil con márgenes de tolerancia para variaciones en las condiciones de origen, por leves que éstas sean.

Así, el problema fundamental reside en cómo hacer inteligible una restricción de las relaciones modales de accesibilidad, si tales relaciones de accesibilidad están dando cuenta de un ámbito de hechos o estados de cosas constitutivos de lo real. Cuando se concibe el ámbito de lo modal como una región dotada de legitimidad ontológica, entonces lo razonable es pensar que las relaciones de accesibilidad por las que quedan delimitados los ámbitos de lo necesario y lo posible son transitivas. Lo posible desde lo posible es posible. ¿Por qué sucede esto? Porque cuando se concibe el ámbito de lo modal como un ámbito ontológicamente objetivo, entonces debe concebirse que establece el dominio del tipo de desarrollos que podría mostrar el conjunto de entidades existentes, con independencia de lo que nosotros creamos que pueden ser tales desarrollos. La totalidad de mundos posibles queda definida por la totalidad de posibles desarrollos de las naturalezas de las entidades actualmente existentes. En la concepción medieval según la que se concibió originalmente la idea de un "mundo posible", se estaba representando la totalidad de posibles modos en que Dios podría haber ejercitado su libertad creadora. La modalidad ontológica y las relaciones de accesibilidad que rigen entre estas posibilidades siguen las propiedades del tipo de vinculaciones causales que se dan entre las entidades actuales y los desarrollos posibles que se siguen de la naturaleza de tales entidades. Pues bien, las relaciones causales son transitivas. Si hay una relación causal entre $A$ y $B$, y hay una relación causal entre $B$ y $C$, entonces hay una relación causal entre $A$ y $C$. Si hay algo así como una modalidad metafísica u ontológica, diferente de la modalidad epistémica, debe ser una modalidad con relaciones de accesibilidad transitivas. ${ }^{22}$

¿Cuál es la motivación, entonces, para introducir una restricción en la transitividad de tales relaciones de accesibilidad? La motivación que guía al

\footnotetext{
${ }^{22}$ Nótese que esta concepción de un dominio modal ontológicamente objetivo no es la concepción llamada "realista" de David Lewis.
} 
defensor de una necesidad de origen débil es el hecho de que sin la restricción no podrían respetarse los umbrales de tolerancia; en otras palabras, parece que se requerieren tales restricciones para la permanencia de lo que es imaginable por el sentido común. Se trata de una restricción que está motivada por una fuente esencialmente "epistémica", esto es, se toma aquí 'posible' como aquello que no resulta impedido aparentemente por nada de lo - según creemos- efectivo. Lo que el sentido común está inclinado a imaginar se constituye en juez de las estructuras ontológicas de la modalidad, y esto no es aceptable, si realmente se pretende respetar un ámbito ontológico objetivo de lo modal. No deben introducirse restricciones en la estructura ontológica de lo modal, tal como razonablemente podemos comprender esta estructura como un ámbito independiente y objetivo de realidad, simplemente por lo que el sentido común se siente inclinado a imaginar. Este tipo de limitaciones podrían ser aceptables si se adoptase una perspectiva antirrealista sobre lo modal, esto es, si de alguna manera se concibiese que el ámbito de los hechos modales está constituido fundamentalmente por proyecciones de nuestra subjetividad. Si así fuese el caso, entonces no habría dificultad en hacer de nuestros poderes imaginativos los soberanos de lo necesario y lo posible. Sin embargo, la idea de necesidad "metafísica" es exactamente el rechazo de esta concepción.

Nótese aquí, en efecto, que si se concibe la "posibilidad" en un sentido epistémico como lo "concebible" o "imaginable", entonces es obvio que la transitividad se quiebra, pues yo podría concebir una situación posible en la que se den ciertas circunstancias y en esas circunstancias posibles se podría concebir otra situación posible que, sin embargo, en las circunstancias actuales yo sería incapaz de concebir. Por ejemplo, en el siglo XIII podría concebir la posibilidad de construir una máquina calculadora; pero también podría ser incapaz de concebir (por falta de imaginación) lo que podría ser perfectamente imaginado mil años después, una vez que ya existan máquinas calculadoras funcionando, como podría ser una sociedad de inteligencias artificiales que dominase el planeta y a todos los seres humanos. El mundo posible $w_{i}$ es tal que en él es posible imaginar que los hombres usáramos máquinas calculadoras. Ese mundo posible $w_{i i}$ donde se usan máquinas calculadoras es tal que desde él es concebible otro mundo posible $w_{i i i}$, donde las computadoras dominen el planeta, pero no se sigue que sea concebible desde $w_{i}$ el mundo posible $w_{i i i}$. Típicamente, la transitividad de las relaciones de accesibilidad se quiebra cuando se trata de una modalidad "epistémica" que tiene que ver con lo que es concebible o imaginable según nuestras capacidades cognoscitivas. ${ }^{23}$ Es obvio que el

${ }^{23}$ Considérese además que no sirve aquí introducir una "concebidora ideal" o algo parecido. Si una concebidora ideal $A$ puede concebir la posibilidad de que exista una concebidora ideal $B$ que concibe, a su vez, que $p$, entonces parecería plausible pensar que la concebidora ideal puede concebir que $p$. Al parecer, en este caso se podrían mantener las relaciones de 
hecho de que toda modalidad epistémica no sea transitiva no implica que toda modalidad en la que las relaciones de accesibilidad no sean transitivas será epistémica. La cuestión, sin embargo, es que aun así se hace necesario para el defensor de una modalidad sin accesibilidad transitiva explicar con precisión cuál es el fundamento para introducir tal restricción si no es una apelación -implícita, si se quiere- a una concepción de la modalidad en la que ésta queda dependiendo de las vicisitudes que pueden acaecerle a nuestras habilidades imaginativas. Y aquí no se aprecia ningún otro fundamento razonable que no sea esta concepción antirrealista.

Si ha de mantenerse la idea de que el ámbito de lo modal constituye un dominio ontológico objetivo, entonces debemos estar dispuestos a asumir las consecuencias que se derivan de la postulación de semejante ámbito ontológico. Estas consecuencias pueden chocar con creencias generalmente aceptadas desde un punto de vista prefilosófico y previo a toda discusión racional, pero las creencias generalmente aceptadas no son el criterio de lo real y lo existente en cielos y tierra. Se sigue de la tesis general sobre la naturaleza de una modalidad ontológica que las relaciones de accesibilidad deben concebirse transitivas. Entonces, esto es una buena razón para reformar en un aspecto esencial las creencias populares sobre la posibilidad de hacer pequeñas variaciones en las condiciones de origen de un objeto sin afectar su identidad. Por el contrario, si la intuición popular sobre la posibilidad de variaciones pequeñas en las condiciones de origen de un objeto fuera a tomarse como el canon de lo modal, entonces se impondrán restricciones sobre lo que razonablemente cabe suponer que es un dominio ontológico objetivo, de acuerdo con lo que el ciudadano común cree que es compatible con su visión ontológica rudimentaria. Se está haciendo del ámbito de lo modal, básicamente, una criatura de nuestra imaginación. En estas condiciones, lo razonable es reformar y mejorar las intuiciones ontológicas rudimentarias y vulgares, o sustituirlas por algo más refinado. En resumen, al poner una atención suficientemente cuidadosa a la concepción de la modalidad como un dominio ontológico objetivo se hace inadmisible imponer las restricciones que se requerirían

accesibilidad transitivas como regla general. No obstante, el problema es que sólo se logran mantener tales relaciones de accesibilidad transitivas si las concebidoras son "ideales" en efecto; esto es, se supone que son sujetos (o máquinas) que no se cansan, que nunca olvidan, que no cometen errores por desatención, que no cometen errores de coherencia en sus creencias, que creen todo aquello que se sigue lógicamente de aquello que creen. Y ningún sujeto real que concibe, ni ninguna máquina que concibe son "ideales" en este sentido. Como todo sujeto o máquina concebidora real puede cometer errores de todo tipo, entonces no vale de manera general el principio de transtividad para las relaciones de accesibilidad entre las posibilidades concebidas por tales sujetos o máquinas. Es obvio que la concepción de una "concebidora ideal" no proviene de la noción de un sujeto o máquina que conciba, sino de las nociones modales ontológicas que se utilizan como patrón de medida para dotar de contenido la "idealidad" a la que debe adecuarse una "concebidora ideal". 
para hacer operativa la necesidad de origen débil. Esto significa que, si hay necesidad de origen, debe haber necesidad de origen fuerte y que las verdaderas opciones teóricas en disputa en lo que concierne a esta cuestión son esta necesidad de origen fuerte o simplemente contingencia de origen. $^{24}$

\section{BIBLIOGRAFÍA}

Forbes, G., "Origin and Identity", Philosophical Studies 37, 1980, pp. 353-362

—. The Metaphysics of Modality, Clarendon, Oxford, 1985.

— - "In Defense of Absolute Essentialism", en P.A. French, T.E. Uehing, Jr., y H.K. Wettstein (comps.), Studies in Essentialism (Midwest Studies in Philosophy, vol. 11), University of Minnesota Press, Minneápolis, pp. 3-31.

—_, "A New Riddle of Existence", Philosophical Perspectives, vol. 8, Logic and Language, ed. J. Tomberlin, Ridgeview, Atascadero, 1994, pp. 415-430.

— - "Origins and Identities", en A. Bottani, D. Giaretta y M. Carrara (comps.), Individuals, Essence and Identity. Themes of Analytic Metaphysics, Reidel, Dordrecht, 2001, pp. 319-340.

Hawthorne, J. y T. Szabó Gendler, "Origin Essentialism: The Arguments Reconsidered", Mind, vol. 109, 2000, pp. 285-298.

Kripke, Saul, Naming and Necessity, Harvard University Press, Cambridge, Mass., 1980. [Versión en castellano: El nombrar y la necesidad, trad. Margarita M. Valdés, Instituto de Investigaciones Filosóficas-UNAM, México, 1995.]

Lewis, D., "Counterpart Theory and Quantified Modal Logic", en Philosophical Papers Volume I, Oxford University Press, Oxford, 1983, pp. 26-46.

- On the Pluralilty of Worlds, Blackwell, Oxford, 1986.

Noonan, H., "The Necessity of Origin", Mind, vol. 92, 1983, pp. 1-20.

Robertson, T., "Possibilities and the Arguments for Origin Essentialism", Mind, vol. 107, 1998, pp. 729-749.

—_, "Essentialism: Origin and Order", Mind, vol. 109, 2000, pp. 299-307, en especial, pp. 301-302.

Salmon, N., Reference and Essence, Blackwell, Oxford, 1982.

_- "Modal Paradox: Parts and Counterparts, Points and Counterpoints", en P.A. French, T.E. Uehing, Jr., y H.K. Wettstein (comps.), Studies in Essentialism (Midwest Studies in Philosophy, vol. 11), University of Minnesota Press, Minneápolis, pp. 75-120.

_- "The Logic of What Might Have Been", The Philosophical Review, vol. 98, no. 1, 1989, pp. 3-34.

Recibido el 3 de agosto de 2004; aceptado el 24 de febrero de 2005.

${ }^{24}$ Este trabajo ha sido redactado en ejecución del proyecto de investigación FIL00303 (FAI, Universidad de los Andes, Chile). Se presentó una versión preliminar en el IV Encuentro de Filosofía e Historia de la Ciencia del Cono Sur organizado por la Asociación de Filosofía e Historia de la Ciencia, Buenos Aires, del 22 al 25 de marzo de 2004. Agradezco especialmente los comentarios que recibí en esa ocasión de Wilfredo Quezada y Guido Vallejos. Agradezco también las sugerencias y observaciones de un evaluador anónimo de la revista Diánoia. 